\title{
LA MATEMÁTICA ESCOLAR COMO LENGUAJE. NUEVAS PERSPECTIVAS DE INVESTIGACIÓN Y ENSEÑANZA*
}

\author{
ROJANO, $T$. \\ Departamento de Matemática Educativa del Cinvestav-IPN, México.
}

\section{SUMMARY}

In this article we have made a collection of the focuses and trends in the research on school mathematics considered as a language, which has characterized the research in the $80 \mathrm{~s}$ and the beginning of the $90 \mathrm{~s}$-in contrast with the $70 \mathrm{~s}$ decade, when the construction of concepts was thought of as the central objective of studying. We also deal -as a matter inherent to research - with the topic of the possible didactic implications that such focus can entail.

\section{INTRODUCCIÓN}

Con el surgimiento del álgebra simbólica en el siglo xvI, nace un lenguaje propio para la matemática, una de cuyas características es la de ser un lenguaje que se autoexplica, esto es, en él no sólo es factible expresar los teoremas sino también demostrarlos.

Pero no siempre el álgebra ha tenido esa autonomía. Durante mucho tiempo, el lenguaje natural y la geometría fueron medios imprescindibles de expresión y validación de las verdades algebraicas, y la evolución simbólica del álgebra puede interpretarse actualmente como una trayectoria de su gradual independencia de dichos medios hasta constituirse en un sistema de signos «autocontenido». Esta presentación final de la matemática como un lenguaje autosuficiente y formal es la que en un tiempo trató de comunicarse a los estudiantes mediante la enseñanza escolar; ello condujo a una profunda crisis en este nivel. Hoy en día, y en algunos casos a raíz de la mencionada crisis educativa, se cuenta ya con numerosos trabajos de investigación desarrollados en los últimos veinte años, los cuales tratan de clarificar la naturaleza del lenguaje matemático y los pormenores de su apropiación.

En el presente artículo se hace un recuento de enfoques y tendencias en la investigación sobre el aprendizaje del lenguaje de las matemáticas que han caracterizado las investigaciones de los años 80 e inicios de los 90 , y se aborda también, como cuestión inherente a la investigación, el tema de las posibles implicaciones didácticas a que pueden dar lugar tales enfoques.
Los casos y ejemplos aquí tratados se refieren en su mayoría al lenguaje algebraico, debiđo funđamentalmente a dos razones: la primera es que, por ser el álgebra simbóilica el lenguaje básico de la matemática, buena parte de los trabajos desarrollados en la línea la matemática como lenguaje se enfocan específicamente al álgebra; y la segunda tiene que ver con el hecho de que mi experiencia personal en el campo de la investigación es mucho más cercana a la didáctica del álgebra que a cualquier otro tema de la educación matemática. Lo anterior no quiere decir que no merezca la pena ampliar la panorámica que se intenta dar en este escrito incluyendo ejemplos de la geometría, la aritmética o el cálculo. Pero, a pesar de esta especie de parcialización del tema, creo que el asunto de los actuales cambios en los objetos de análisis, en la metodología de investigación y en las perspectivas de enseñanza de las matemáticas puede ser abordado, en sus aspectos esenciales, desde el caso particular, pero significativo, del lenguaje algebraico.

\section{UNA VISIÓN CONCEPTUALISTA DE LA EDUCACION MATEMATICA}

Los acercamientos constructivistas en la indagación de los fenómenos del aprendizaje matemático, provenientes principalmente de la corriente de la psicología genética de Piaget, imprimieron a la tarea investigativa en 
educación matemática de la década de los 70 un sello particular, el de considerar la construcción de conceptos como su objeto central de estudio. Así, nos encontramos en ese período con una gran producción de trabajos sobre, por ejemplo, la construcción del concepto de número, de la noción de variable o de la noción de función (entre muchos otros, realizados sobre una diversidad de conceptos matemáticos) y se advierte en los reportes correspondientes un énfasis en señalar las dificultades que entrañan dichas construcciones. El enfoque constructivista trascendió no sólo al ámbito de la investigación en psicología de las matemáticas, sino que también alcanzó el campo de la didáctica, de la enseñanza y del điseño y desarrollo curriculares en matemáticas.

Lo anterior explica, en parte, la puesta en marcha de reformas del currículo que trataban de romper con los acercamientos estructuralistas a la enseñanza de las matemáticas, presentes en muchos programas de estudio de la década anterior (la de los años 60).

Uno de los efectos más notorios sobre las mencionadas reformas educativas se observa en el tratamiento específico y muy detallado de temas o nociones matemáticas que la investigación demostraba que requerían de tiempos didácticos muy prolongados y de ciertas condiciones de maduración cognitiva de los alumnos. Así, aparte de las ventajas que representa el tener en cuenta los obstáculos y dificultades inherentes al aprendizaje de un cierto concepto matemático (como, por ejemplo, la ventaja de renunciar a una visión "eficientista» de la enseñanza y en su lugar propiciar la evolución de los sujetos hacia la construcción de dicho concepto), el enfoque conceptualista se enfrenta también con el problema de acoplar los mencionados tiempos didácticos a los tiempos reales de los ciclos escolares y, por tanto, también enfrenta la necesidad de una reconsideración del conjunto de conocimientos matemáticos básicos que todo individuo debiera poseer. En algunos casos, el resultado de una posición como la anterior fue una tendencia a la postergación de temas enseñados tradicionalmente en la escuela elemental al ciclo escolar posterior (tal fue el caso de la enseñanza de las fracciones en el currículo francés).

Volviendo al ámbito de la investigación, en el panorama que podía apreciarse todavía durante la década pasada, predominaba la preocupación por conocer a fondo los procesos de construcción y aprehensión de determinados conceptos matemáticos. Para algunos de éstos, se elaboraron modelos teóricos ex profeso, como, por ejemplo, el modelo de construcción del número racional de Kieren (1988). O se hicieron adaptaciones del modelo piagetiano de las etapas de desarrollo de los conceptos, como el trabajo de Küchemann y Booth (en el campo del álgebra) sobre la interpretación de los símbolos literales por parte de los alumnos (Booth 1984 y Küchemann 1981) o el trabajo teórico de Van Hiele, desarrollado para nociones geométricas (Van Hiele 1987). O se conformaron líneas de investigación que incorporan elementos de la cpistemología genćtica y de la historia de los conceptos matemáticos a fin de poder identificar las principales dificultades y obstáculos didácticos de la construcción de un determinadoconcepto-por ejemplo, el trabajode Brousseau sobre los números decimales (Brousseau 1981)-. S6́lo por mencionar algunas de las diversas modalidades de investigación educativa con una aproximación constructivista.

Aparejado a la tendencia ya señalada en las investigaciones desarrolladas a fines de los años 70 y durante los 80 , empezó a surgir el interés por parte de algunos autores en estudiar los aspectos semántico y sintácticode la matemática, a fin de poder explicar las observaciones hechas acerca de las interpretaciones y usos que los estudiantes dan a los símbolos matemáticos. Con el tiempo, este interés fue en aumento, dando lugar a un cambio significativo en la investigación en educación matemática, la cual pasa de mirar la matemática escolar como redes de conceptos que el sujeto va a construir a través de su experiencia en la escuela a considerarla como un lenguaje. Con este giro, los sujetos son vistos como usuarios potenciales del lenguaje matemático, y la enseñanza, como el medio que debe propiciar el aprendizaje de dicho lenguaje.

\section{LA MATEMÁTICA COMO LENGUAJE. UNA NUEVA PROBLEMÁTICA}

La nueva tendencia a relacionar el aprendizaje de la matemática con los procesos de adquisición y uso de dicho lenguaje, más que con su construcción concepto a concepto, conduce a reformulaciones importantes acerca de los objetos de estudio y de los fenómenos que hay que observar en el campo de la investigación. Estos replan. teamientos varían de unos autores a otros, ya que la diversidad de trabajos que se pueden englobar en la tendencia mencionada corresponden, a su vez, a una variedad de enfoques. Por cierto que muchos de tales enfoques parten también de una visión constructivista del conocimiento matemático; lo cual quiere decir que la matemática como lenguaje no es necesariamente una concepción que se contraponga a las concepciones enraizadas en el constructivismo.

En las secciones subsiguientes, se hará referencia a trabajos desarrollados en la línea del aprendizaje y enseñanza del lenguaje matemático y se intentará completar un panorama de tendencias en la investigación actual alrededor de una problemática que se encuentra aún en evolución.

\section{LA MATEMÁTICA Y OTROS LENGUAJES}

\section{E] carácter formal de las matemáticas}

Una de las aportaciones en la dirección de clarificar las dificultades de la apropiación del lenguaje matemático por los estudiantes es el formidable intento de caracterización del lenguaje matemático (algebraico) realizado por Hans Freudenthal en el capítulo 16 de su libro Didactical Phenomenology of Matematical Structures (1983), en el que las precisiones acerca de las dificultades del aprendizaje del álgebra se hacen en términos comparativos con aquéllas que enfrentan los sujetos al 
aprender la lengua materna. En este trabajo, las piezas conceptuales dispuestas de una cierta manera por las investigaciones de los años 70 se recomponen, y se hacen plausibles otras explicaciones de la presencia de tales dificultades. Por ejemplo, la presencia y posibjlidad de rectificación de los llamados errores de sintaxis algebraica, como el de la sobregeneralización de reglas o propiedades (uno de los ejemplos más populares de este tipo de sobregeneralizaciones es el de la distribución lineal de un operador respecto a otro, como en $\left.(a+b)^{2}=a^{2}+b^{2}\right)$, se explica por el hecho de que el álgebra simbólica es un lenguaje cuyo uso está restringido al aula, en contraste con el uso consuetudinario del lenguaje natural, el mismo uso que permite que, por ejemplo, el error típico en niños pequeños de conjugar como regulares algunos verbos que no lo son tenga una rectificación a fuerza de su uso y retroalimentación frecuentes.

De acuerdo con este autor, una explicación más precisa de lo anterior tiene su fundamento en una de las diferencias esenciales entre el lenguaje vernáculo y el matemático; a saber, el hecho de que este último cuenta con la fuente más importante de formalización progresiva: la construcción algorítmica del vocabulario. Estos rasgos algorítmicos no están ausentes en el vocabulario y sintaxis de cualquier lenguaje, pero son incidentales y asistemáticos (señala Freudenthal). Así, los plurales, el tiempo pasado, los términos comparativos, etc. del lenguaje natural no se acercan nj remotamente a la estructura sistemática de los numerales en la matemática.

Una comparación semejante la lleva a cabo el autor con respecto al lenguaje aritmético. En ambas comparaciones, enfatiza el carácter formal del lenguaje algebraico (aunque reconoce a la aritmética ciertos rasgos formales en el terreno de las operaciones elementales). Apunta que esta característica de ser un lenguaje formal impide la reducción del álgebra a cualquiera de esos dos casos (el de la aritmética o el del lenguaje natural), sobre todo en lo que a procesos de comunicación se refiere. Esto es, mientras que los errores que se cometen en la aritmética o en la conjugación de verbos irregulares en el espafiol tienen posibilidades de rectificación a largo plazo, debido a que el uso frecuente permite una retroalimentación también frecuente a partir de contextos muy familiares para los sujetos, como son el contexto numérico -en el caso de la aritmética-, y el contexto de la comunicación cotidiana con usuarios competentes -en el caso del lenguaje natural-, las rectificaciones de los errores que se cometen en el álgebra son poco factibles por esas vías. La razón principal de elto, según lo expresa Freudenthal, es que conjugar un verbo irregular como si fuera regular o colocar un acento escrito sobre una letra equivocada, por to general no da lugar a equívocos y estas faltas pueden ser dispensadas en el lenguaje vernáculo, ya que en este caso con frecuencia los criterios de contenido más que los formales deciden la estructura; en otras palabras, la comunicación no se altera mayormente en su contenido. Sin embargo, en el lenguaje de las matemáticas, el criterio del contenido o significado no es confiable, pues por ejemplo:

5 veces ... 3 más 7 tiene tanto significado como

5 veces $3 \ldots$ más 7 ,

y, si se ha de usar esta expresión, debe estar perfectamente claro lo que ella significa, lo cual se consigue solamente con estrictas reglas de puntuación y de cómo leer una expresión y colocarla entre paréntesis.

Para contrastar con el caso de la lengua vernácula, Freudenthal utiliza el siguiente ejempio tomado del inglés:

\section{Las oraciones}

\section{there were aged ladies and children in the bus y}

\section{there were aged ladies and gentlemen in the bus}

son sintácticamente equivalentes, sin embargo, aplicando un criterio de contenido, en la primera el adjetivo aged sólo puede referirse a ladies, mientras que, en la segunda, puede estar afectando tanto a ladies como a gentlemen.

Las reflexiones de Freudenthal en torno a las diferencias y similitudes del lenguaje algebraico con la lengua materna y la aritmética sugieren dimensiones de análisis de dicho lenguaje que contemplen sus aspectos sintácticos y semánticos. A este respecto pueden mencionarse una serie de investigaciones que examinaremos en el apartado siguiente y que definen sı problemática en términos de símbolos y lo que representan o, como en el caso de Vergnaud, en términos de la naturaleza de ser indisociables, uno de otro, del significado y el significante (Vergnaud 1981).

\section{Conocimiento matemático y habilidades lingitísticas}

La perspectiva que adopta Vergnatud permite una concepción constructivista en un sentido más amplio (que la concilia con la necesidad de tomar en cuenta el papel del lenguaje): «El conocimiento es activamente construido por el sujeto organizador quien, en un proceso adaptativo e interactivo con su medio ambiente, organiza su mundo de experiencias» (Vergnaud 1987). Dicha concepción se refiere no solamente a la adquisición del conocimiento matemático, sino también al desarrollo de las habilidades linguísticas mismas. Así, según lo indica C. laborde en el capítulo 3 de Mathematics and Cognition (Laborde 1990), ello significa que, desde una perspectiva psicolinguística, cl objeto que ha de ser estudiado no es el discurso mismo, sino el discurso como resultado de Ia actividad conceptual en un contexto dado y en un ambiente social determinado.

La anterior reformulación desde una aproximación constructivista ya apunta hacia la delimitación de objetos de estudio diferentes de aquéllos de la corriente conceptualista (también basada en una noción constructivista del conocimiento) e imprime a la tarea investigativa necesidades de renovación teórica y metodológica. Siguiendo a Laborde (1990), habría dos preguntas cen- 
trales que las nuevas investigaciones debieran intentar responder:

- ¿Cuáles son los problemas debiđos a las especificidades del lenguaje matemático con los que los estudiantes se enfrentan al leer o escribir matemáticas?

- ¿De qué manera la enseñanza puede ayudar a desarroliar en los estudiantes habilidades para leer o hacer formulaciones en lenguaje matemático?

Una necesidad metodológica de la primera de las preguntas es la realización de investigación diagnóstica basada en hipótesis psicológicas y lingüísticas, mientras que la segunda requiere de experimentación en el salón de clases basada en hipótesis sobre la naturaleza de la adquisición del conocimiento en el entorno escolar y sobre los factores de desarrollo de dicho conocimiento.

El enmarque teórico en el que cobran sentido los planteamientos anteriores (tanto las preguntas de investigación como las necesidades metodológicas) está delineado por el acercamiento constructivista del conocimiento, que se establece en un sentido más amplio (como se ha señalado en un párrafo anterior) y que toma en cuenta los elementos fundamentales de las situaciones de aprendizaje escolar, a saber: los contenidos de enseñanza, el estudiante desde un punto de vista cognitivo y social, y el maestro.

De manera específica, las investigaciones que se apegan a las consideraciones expuestas por Laborde abordan el tema del tránsito de unas a otras formas del lenguaje. Comocasos particulares Laborde trata: El Lenguaje Natural y las Formas Notacionales en la Aritmética, y El Tránsito de la Aritmética al Álgebra (específicamente la Influencia del Lenguaje Natural en dicho tránsito).

En el primer caso, pueden citarse ejemplos que corresponden a resultados obtenidos en estudios realizados por Conroy (1981), cuyo propósito es indagar si hay una jerarquía en las diferentes formas de lenguaje utilizadas para las cuatro operaciones elementales. Una de las conclusiones a las que se llega es que, en efecto, hay jerarquías que reflejan el lenguaje del salón de clases pero que también existen algunas estructuras del lenguaje peculiares a la matemática que el niño tiene que aprender, ya que dichas estructuras no tienen lugar con frecuencia en el lenguaje natural. Particularmente, se tiene la evidencia de que en el idioma inglés, la decodificación de sonidos es mucho más difícil que la decodificación de símbolos. Por ejemplo, las instrucciones orales para sumar, usando términos como greater than o more than y la instrucción oral less than resultaron ser, en este estudio, las formas de lenguaje más difíciles para estudiantes de segundo y tercer grado de primaria.

En relación con el pasaje de la aritmética al álgebra y la influencia del lenguaje natural, investigadores como Clement (1982) y Cooper (1984) han señalado factores linguíisticos provenientes del lenguaje natural que afectan la traducción de un enunciado dado en este lenguaje al lenguaje algebraico. Un ejemplo ampliamente conocido es lo que sucede con el problema de elos estudiantes y los profesores»: muchos estudiantes de la escuela pre-uni- versitaria escriben la ecuación $6 S=P$ cuando se les pide que traduzcan al álgebra el enunciado «Hay seis veces tantos estudiantes como profesores».

Del mismo modo, Norman (1987) muestra con otro ejemplo cómo la sintaxis del lenguaje natural podría afectar la percepción de la estructura algebraica por parte del estudiante. El ejemplo es el siguiente:

En un viaje, Juan manejó un total de 50 millas en una hora. Una parte del viaje la recorrió a una velocidad de 35 millas por hora. ¿A qué velocidad recorrió la otra parte?

En ese problema, la tercera parte de un grupo de estudiantes para profesor de escuela primaria dio como respuesta que Juan manejó a una velocidad de 15 millas en la segunda parte del viaje. Es decir, propusieron una respuesta aditiva. Norman interpreta este hecho pensando que aquí la sintaxis parece imponer una estructura aditiva al lenguaje algebraico. Norman hace notar con este ejemplo que los estudiantes encuentran la scmántica del enunciado algebraico en el marco de referencia del lenguaje natural y, especialmente en este caso particular, en la sintaxis del lenguaje natural.

Laborde (1990) elabora algunas conclusiones a partir de las investigaciones referidas, una de ellas apunta en la dirección de que los aspectos cognitivo y linguístico intervienen simultáneamente en la comprensión y uso de los diferentes tipos de formulaciones: la semántica de una formulación es construida por el estudiante por medio de sus representaciones mentales y de los rasgos lingüísticos de la formulación. Sobre la base de lo anterior, que parece indicar que cl lenguaje natural representa un papel determinante, propone realizar estudios en profundidad sobre los procesos mediante los cuales un individuo construye un significado o utiliza un modo de formulación, y la dependencia del contexto y contenido matemáticos que subyacen a dicha formula. ción. Una razón de esta necesidad de profundización es que (según Laborde) los modelos formales de descripción de los sistemas lingüísticos no son suficientes para explicar tales procesos.

\section{EN LA TRANSICIÓN DE LA ARITMÉTICA AL ÁLGEBRA}

Dentro de la misma vertiente de investigaciones que se ocupan de la interacción del lenguaje algebraico con otros lenguajes, se encuentra el estudio realizado por Filloy y Rojano (1991), en el cual las respuestas de estudiantes de 12 a 13 años de edad a problemas de traducción del lenguaje natural al álgebra y viceversa son analizados en el marco de una serie de tendencias cognitivas presentes en el aprendizaje de conceptos «más abstractos» (Filloy 1991 y 1993).

El momento de la entrevista clínica, en cl estudio mencionado, fue elegido para poder observar las tensiones existentes entre los significados atribuidos a los conceptos algebraicos elementales en vías de construcción y los significados provenientes de los campos conceptuales 
aritmético y del pre-álgebra, sobre los cuales se erige el nuevo conocimiento, el algebraico. Dicha tensión, afirman Filloy y Rojano, es el resultado de la necesidad de dotar de un nuevo sentido (a través del uso) a las nuevas operaciones y conceptos, lo que a su vez dotará de nuevos significados a las expresiones algebraicas representadas por los mismos signos (de la aritmética) o versiones más elaboradas de elios.

Cabe hacer notar que, en términos de instrucción, el momento de observación señalado se corresponde con el momento en que los alumnos habían recibido enseñanza en pre-álgebra y apenas habían sido introducidos al álgebra elemental con el tema de resolución de ecuaciones lineales y problemas verbales asociados. El siguiente ejemplo ilustra la manera en que la lectura de los símbolos algebraicos, en este momento de transición de un tipo de lenguaje a otro, está permeado por los significados atribuidos anteriormente a dichos símbolos:

En un bloque de preguntas de la entrevista se les pedía a los alumnos que interpretaran expresiones como: $\frac{(a+b)}{2}, a b, 3 a b, a^{2}$. En la expresión $\frac{(a+b)}{2}$, la respuesta típica fue una lectura «textual» de la misma, «a más $b$ sobre dos», acompañada de una referencia verbal a dimensiones de figuras geométricas «ideales», por ejemplo: «base más altura sobre dos», acompañada también esta lectura de la necesidad de asignar valores específicos a las letras, a fin de obtener un resultado y de cerrar la expresión inventada por los propios alumnos. Un comportamiento análogo, aunque no tan completo, ya había sido reportado por Booth (1984) y Collis (1975). En este caso puede notarse la influencia de los significados que son atribuidos en las fórmulas geométricas a los símbolos literales, así como la necesidad aritmética de completar un proceso operativo (aquí se trata de la operación suma).

Entre las respuestas más sofisticadas a esta pregunta, y con una fuerte influencia del pensamiento pre-algebraico del niño está la elaboración de una ecuación o igualdad a partir de la expresión $\frac{(a+b)}{2}$ y la substitución numérica posterior para alguna de las letras, por ejemplo: $\frac{(a+b)}{2}=10$ se convertía en $a+3=10$.

Booth y Collis describen estas observaciones como una tendencia cognitiva consistente en el regreso a situaciones más concretas ante la ocurrencia de una situación de análisis. En este caso, los niveles de interpretación, en que los referentes geométricos están asociados con ellos, corresponden a lo que aquí se está llamando «situaciones más concretas». Como consecuencia, se menciona en el trabajo referido la necesidad de utilizar modelos cognitivos más elaborados, en los cuales las tendencias cognitivas, como la recién citada «regreso a situaciones más concretas», aparezcan como manifestaciones naturales de los procesos de abstracción que se producen cuando el sujeto está aprendiendo a ser un usuario más competente de sistemas de signos como el algebraico y otros intermedios, que han sido socialmente producidos y que los sujetos en observación tienen que dominar a través de los modelos de enseñanza usuales.

Más adelante, se presentan algunos acercamientos té. ricos recientemente desarrollados, los cuales podrían dar cuenta de observaciones empíricas, como la reseñada en los párrafos anteriores, para las cuales las explicaciones elaboradas desde los marcos teóricos conceptualistas, disponibles hasta el momento, parecen insuficientes.

\section{ASPECTOS SEMÁNTICO Y SINTÁCTICO DEL LENGUAJE MATEMÁTICO}

\section{Algunos análisis y trabajos empíricos}

Los aspectos sintáctico y semántico de la matemática se convirtieron en centro de atención de las investigaciones recientes, a raíz de las observaciones realizadas en estu* dios que incluían tareas de traducción de un cierto lenguaje o tipo de representación al lenguaje matemático o viceversa. Un ejemplo típico son los estudios sobre la resolución de problemas en matemáticas, pues los procesos estudiados incluyen fases de interpretación y traducción, por lo general, entre el lenguaje natural y la matemática.

En los estudios llevados a cabo en relación con la resolución de problemas algebraicos verbales, un común denominador es la ausencia de métodos algebraicos en las respuestas de alumnos entre 12 y 16 a ̃ŭos de edad. Puede haber múltiples explicaciones de este hecho, pero una de las causas que hay que señalar es que los estudiantes no logran integrar los dos dominios de su conocimiento, conformados, por un lado, por el manejo sintáctico del álgebra y, por otro, por la resolución de problemas. Esta integración está condicionada por la posibilidad de construir una semántica de los símbolos y operaciones algebraicas, ligada a las situaciones que estarán presentes en los enunciados de los problemas que hay que resolver algebraicamente. Esto es, tal integración implica una interacción armónica entre la sintaxis del álgebra y los significados asignados a ella. De aquí surge la necesidad de ahondar en el estudio de dicha interacción, ya que los esquemas de análisis anteriores parecen ser insuficientes para clarificar su naturaleza.

Desde principios de la década pasada, varios autores han tratado de manera explícita los temas de la semántica y la sintaxis de la matemática. Quisiera referirme al caso específico de los artículos que aparecen en el número 3 del año 1982 de la revista Visible Language, númeroespecial dedicado a la «Comprensión del Simbolismo de las Matemáticas", y en el que los autores abordan diferentes aspectos de dicha temática.

\section{Tendencias semánticas y tendencias sintácticas}

Un tema poco abordado por las investigaciones sobre la semántica y la sintaxis algebraicas es el de las actitudes o tendencias individuales existentes en los alumnos 
hacia "preferir" ciertos métodos de resolución, los cuales varían en un amplio espectro que va desde los métodos más operativos y algorítmicos hasta los más semánticos y analíticos. En el estudio clínico Operación de la Incógnita, desarrollado por Filloy y Rojano (1989), se reporta la presencia de este tipo de tendencias en estudiantes que se inician en el estudio del álgebra. Específicamente se analizan dos casos de tendencias extremas y antagónicas: una niña, Valentina, con tendencia semántica y una niña, Matilde, con una marcada tendencia sintáctica.

La observación se realizó mediante entrevista clínica individual y se trataba de enseñar al sujeto, por primera vez, la resolución de ecuaciones lineales con ocurrencias de la incógnita en ambos lados de la igualdad. Para ello se utiliz6 un modelo «concreto». El modelo de enseñanza en cuestión es un modelo geométrico, en el que la equivalencia de áreas es el punto fundamental de apoyo para realizar las acciones uconcretas» que resuelven una ecuación dada (Filloy y Rojano 1989). A Valentina y Matilde se les proporcionaron solamente elementos básicos del modelo para que ellas hicieran un uso «libre» del mismo para resolver la ecuación. La tendencia sintáctica de Matilde se manifest 6 por medio de un desprendimiento muy rápido del modelo y por la abstracción de las acciones realizadas en el contex to concreto hacia el nivel sintáctico (acciones con los elementos de la ecuación); en una lista de doce ecuaciones para resolver, Matilde sólo utilizó el modelo en los dos primeros casos y siempre demandó conocer las reglas operativas. Valentina mostró su tendencia semántica con un apego al modelo durante toda la secuencia de ejercicios: aun en aquellos casos de ecuaciones en que la modelación por medio de áreas era muy compleja, ella hizo adaptaciones inesperadas y en algunos casos correctas del modelo geométrico. Aun bajo demanda del entrevistador de empezar a realizar las acciones en la ecuación misma, Valentina siempre prefirió utilizar la semántica del modelo.

Una de las conclusiones más importantes en este estudio, expresada por los propios autores, es que existen fenomenos de abstracción de las acciones en los procesos de modelación concreta, que dependen fuertemente de las tendencias individuales encontradas y que, por lo tanto, es muy riesgoso hacer generalizaciones acerca de la evolución de ciertas operaciones de un nivel «concreto" hacia una forma sintáctica. Esta advertencia cobra sentido en relación con aquellos trabajos que dan un gran peso a la modelación «concreta» o al acceso a fuentes de significado en la construcción de una semántica de los símbolos y conceptos matemáticos. Tal es el caso de la investigación que trataremos en el apartado siguiente.

\section{Modelos teóricos}

Kaput (1987) usa el término "alienación del álgebra» para referirse al devastador problema educativo de la falta de motivación y de la falta de babilidad para aplicar la matemática como una herramienta de interiorización personal y de resolución de problemas. Y señala que el núcleo de esta alienación to constituyen dificultades inherentes al manejo de un sistema simbólico formal, aislado de otro conocimiento que pudiera proporcionar, ya sea retroalimentación informativa respecto a la apropiación de acciones realizadas o un contexto cognitivamente estabilizador de esas acciones.

De nuevo aparece la disociación semántica/sintaxis, y las respuestas pedagógicas que se han tratado de dar al problema de las dificultades del manejo simbólico de la matemática, según lo señala Kaput, consisten en programar para los alumnos aun más ejercitación en dichas maniobras simbólicas (Kaput 1987). Las respuestas al problema, en el nivel de la investigación, provienen de hallazgos de los trabajos de las ciencias cognitivas sobre adquisición de destrezas, los cuales proponen el desarrollo de la estructura del aprendizaje y aplicación de las destrezas de manipulación simbólica, para el diseño de la instrucción. Algunas respuestas de orden tecnológico criticadas por Kaput consisten en aceptar el acercamiento "aislacionista», pero proporcionando ambientes de aprendizaje de destrezas con elementos adicionales que enriquezcan la experiencia manipulativa de símbolos, por medio de representaciones explícitas de trayectorias de pensamiento y cálculo, o por medio de «ayuda inteligente» sobre cómo manipular símbolos, aislados de significados más amplios (entre otros dispositjvos computacionales).

El marco teórico de referencia que el propio Kaput propone, para el caso particular del álgebra, parte deI reconocimiento de la necesidad de considerar un conjunto de lenguajes o representaciones con los cuales comunicar y pensar sobre el lenguaje algebraico. I.os propósitos de este marco referencial son tres (Kaput 1989):

1) proporcionar medios para describir el cntretejido de los lenguajes que conforman el álgebra (expresiones, ecuaciones, tablas de datos, gráficas cartesianas, construcciones híbridas que involucran fragmentos de lenguaje natural, etc.);

2) complementar con una orientación linguiística/representacional la orientación tradicional cognitiva del lenguaje utilizado para describir los fenómenos de aprendizaje y aplicación del álgebra por parte de los estudiantes; $y$

3) proporcionar medios de evaluación de las características de nuevos y potenciales ambientes de aprendizaje y aplicación del álgebra, incluyendo ambientes con elcmentos de apoyo cibernético.

Kaput parte de una acepción amplia de sistemas de representación, que incluye los sistemas familiares de gráficas cartesianas, ecuaciones, etc., así como las representaciones no matemáticas del lenguaje natural y los diagramas o dibujos. Sugiere además que el aprendizaje de la matemática puede mirarse como construcción de significados. Sobre la base de lo anterior, afirma que los significados matemáticos pueden ser establecidos al menos de cuatro maneras. Las tres primeras son:

1) por transformación dentro de un sistema particular de 
representación sin referencia a ninguna otra representación;

2) por traducción entre distintos sistemas matemáticos de representación; y

3) por traducción entre representaciones matemáticas y representaciones no matemáticas.

Kaput señala que ta experiencia repetida con esas tres primeras fuentes deriva en una cuarta fuente de significado, creciente a largo plazo y que aparece a todo lo largo de la matemática, a saber:

4) la consolidación y reificación de acciones, procedimientos y conceptos en objetos fenomenológicos que puedan servir de base para nuevas acciones, procedimientos y conceptos en un nivel más alto de organización (la abstracción reflexiva).

Uno de los supuestos más importantes en este planteamiento teórico es el de negar la existencia de significados absolutos o de fuentes absolutas de significado para la matemática y, en cambio, asumir que los significados se construyen con relación a una representación particular. Por ejemplo, la palabra función no posee un significado absoluto, sino un entretejido de significados que se construyen a partir de las múltiples representaciones de las funciones. Así, según su significado procedimental, la función es un transformador de números, y su correspondiente representación es: $f(x)=\ldots$ Y según su significado relacional, la función es una relación entre números y se representa así: $y=\ldots$.

En el enfoque de Kaput, los objetos o fenómenos estudiados son los actos de representactón, y se parte de la base de hacer una distinción entre las representaciones mentales (medios con los que un individuo organiza su flujo de experiencia) y los sistemas de representación (artefactos linguísticos o culturales materialmente reali. zabłes y compartidos por una comunidad cultural). Una descripción detallada del modelo, así como de sus aplicaciones se encuentra en Kaput (1989).

\section{Más sobre modelos teóricos}

Con una preocupación similar a la del acercamiento teórico de Kaput, en el que las fuentes de significado de la matemática aparecen en primer plano, se encuentran otros trabajos que se basan en nociones provenientes de la semiótica, como la noción de sistemas de signos. Tal es el caso del trabajo de Filloy (1990), quien introduce el concepto metodológico de modelos teóricos locales, en el cual cl objeto de estudio es abordado desde tres componentes interrelacionados:

1) los modelos de enseñanza (por ejemplo, los modelos de enseñanza de los racionales o los modelos para enseñar la resolución de ecuaciones lineales);

2) los modelos de los procesos cognitivos involucrados (por ejemplo, el modelo desarrollado por Goldin para la resolución de problemas (Goldin 1987); y
3) los modelos de competencia formal, los cuales simulan la ejecución competente de un usuario ideal del lenguaje matemático (Matz 1982 y Thompson 1989).

De este modo, en lugar de argumentar en favor de privilegiar cualquiera de estos componentes respecto a los otros, Filloy propone concentrarse en modelos teóri. cos locales adecuados únicamente para fenomenos específicos, pero que contemplen dichos componentes y propongan diseños experimentales ad hoc que clarifiquen las interrelaciones y oposiciones que tienen lugar durante los procesos relativos a cada uno de los tres componentes mencionados.

Además de la noción relativa de modelos locales, otra característica del acercamiento teórico de Filloy es la extensión asignada a la noción de sistemas matemáticos de signos (SMS), pues ésta comprende tanto el significado matemático formal como el pragmático y permite trabajar, además de con los procesos de decodificación de los símbolos, con una teoría de producción de signos que, a su vez, admite el estudio de sistemas de signos intermedios utilizados por el estudiante durante los procesos de enseñanza-aprendizaje. Los SMS, junto con las fucntes de significado pudieran ser puntos de concordancia en los respectivos trabajos de Kaput y Filloy: los diferentes sistemas matemáticos de signos en la propuesta de Filloy se corresponden, en cierta medida, con los sistemas representacionales de Kaput, y, de éstos, las representaciones cognitivas podrían, a su vez, identificarse con el componente de modelos cognitivos en Filloy.

Un rasgo distintivo de la aproximación por medio de los modelos locales es que, en contraste con la búsqueda de un paradigma teórico, aquí el punto de partida es que las suposiciones sobre la naturaleza y límites de la materia y método particulares de estudio, así como lo que cuenta como evidencia, determinan la forma que adoptará el marco local de trabajo, al interpretar fenómenos específicos y al proponer nuevos diseños experimentales, que harán avanzar la teoría a fin de que ésta englobe otras o nuevas evidencias (Filloy 1988).

Pero el interés de marcar similitudes y diferencias entre unos acercamientos y otros obedece, sobre todo, al hecho importante de la presencia de la noción de sentido en aquellos modelos que ponen en juego elementos de la pragmática del lenguaje (que en el caso aquí tratado están específicamente referidos al lenguaje matemático). En el siguiente apartado me referiré a un par de trabajos teóricos, en uno de los cuales, el concepto de sentido resulta fundamental para sus planteamientos.

\section{MODELOS GRAMATICALES. EL ESTUDIO DE LA SINTAXIS}

Los trabajos a los que me refiero en este apartado pertenecen a una línea cuyo interés central lo constituye la sintaxis matemática o, más específicamente, la sin- 
taxis algebraica (considerando que el álgebra simbólica es el lenguaje básico de la matemática).

\section{EI modelo de Kirshner}

La propuesta teórica de Kirshner consiste en un modelo que interpreta las manipulaciones algebraicas como un lenguaje en el sentido de Chomsky (1957). El modelo en cuestión es elaborado a partir de la adaptación de los métodos de la linguística generativa transformacional al estudio del álgebra (Kirshner 1985). La evaluación experimental de esta teoría, más bien predictiva que explicativa, revela detalles del llamado conocimiento estratégico que las teorías con acercamientos conceptuales ocultan. El conocimiento estratégico, por ejemplo, permite que $3 x^{2}$, a pesar de tener dos decodificaciones posibles, $(3 x)^{2}$ y $3(x)^{2}$, se lea de una sola de las maneras.

El modelo gramatical propuesto por Kirshner consiste en modelar las expresiones algebraicas y sus transformaciones, a través de traducciones de las llamadas formas superficiales a las formas profundas y de unas formas a otras en el nivel profundo.

Una de las limitaciones de este modelo, que el propio Kirshner señala, es que, frente a la modelación en la ciencia cognitiva, un acercamiento linguístico formal sólo puede adoptarse para el estudio de un «lenguaje», es decir, para un conjunto de oraciones compuestas de símbolos específicos. Por ello, el paradigma lingiístico no resulta adecuado para el estudio de los procesos de resolución de los llamados problemas verbales o de Ia traducción del lenguaje natural al lenguaje matemático, entre otros fenómenos que son pertinentes para la educación matemática. Sin embargo, el mismo Kirshner afirma que dentro del dominio restringido de las destrezas de la manipulación simbólica, los acercamientos lingüísticos ofrecen ciertas ventajas significativas (Kirshner 1987).

Una de las implicaciones curriculares de este enfoque es señalada por el propio autor y consiste en que el modelo propuesto permite, a través de la investigación básica, un conocimiento preciso de los fenómenos estudiados y ello permite, a su vez, un diseño altamente preciso de las partes del currículo relacionadas con tales fenómenos.

\section{El álgebra es más que sóto conceptos}

En el trabajo doctoral de Drouhard (1992), se expone de manera muy nítida una diferencia que he tratado de establecer desde el inicio de este escrito, a saber, la diferencia entre los acercamientos conceptualistas y los acercamientos psicolingứístcos en la educación matemática. Una consideración de suma importancia en la tesis de Drouhard es que en el álgebra hay ciertos objetos de enseñanza que no son conceptos. Por ejemplo (señala Drouhard) la factorización y la expresion $a x+b$ no son conceptos matemáticos, sin embargo, esta última es una oración bien formada y por tanto es un significante en el álgebra, y llegar a saber si los alumnos les atribuyen alguna significación, y cuál, es crucial para la didácticá del álgebra. Por cllo, este autor plantea como problema básico clarificar qué son las escrituras algebraicas (ya que no son conceptos) y cuáles sus significaciones.

La empresa teórica de Drouhard consiste en poner en evidencia las características principáes de «los modelos estructurados», didácticamente plausibles, de los «sistemas de escrituras simbólicas del álgebra clementaly (Drouhard 1992). Él mismo reformula su propósito en términos tomados del campo de la inteligencia artificial: elaborar los principios característicos de un sistema que modele las prácticas efectivas de los «expertos» en el dominio del cálculo algebraico formal elemental. Al desarrollar su proyecto, Drouhard se basa en dos hipótesis fundamentales: 1) que las escrituras simbólicas del álgebra tienen una estructura, y 2) que la gramática generativa-transformacional de Chomsky es un formalismo adecuado para describir dicha estructura. De este modo, él hace una adaptación del modelo chomskiano al caso de las escrituras simbólicas del álgebra y estudia en detalle estas escrituras y sus transformaciones.

Con esta pieza de trabajo teórico, Drouhard hace una aportación a un conocimiento más preciso del aspecto sintáctico del lenguaje matemático (del álgebra simbólica) y complementa el conocimiento producido por los estudios sobre comprensión o construcción de conceptos en el álgebra, pues, como él mismo lo señala, existen objetos de enseñanza del álgebra, cuyas dificultades de aprendizaje no pueden ser descritas en términos conceptuales, ya que dichos objetos no constituyen conceptos.

La parte empírica de este trabajo, así como las nociones de denotación y designación, basadas en las nociones manejadas por G. Frege $(1962,1974)$, permiten reinterpretar producciones sintácticas de los alumnos, a las cuales se solía dar una explicación de corte conceptualista, es decir, eran interpretados como errores conceptuales. El siguiente es un ejemplo de estas reinterpretaciones, escogido por su simplicidad y tomado del trabajo de Baruk (1973):

Se entrevistó a un alumno sobre la producción:

$$
2=\frac{10}{5}=\frac{4+6}{4+1}=\frac{6}{1}:-6
$$

Cuando se trat $\delta$ de hacer ver al alumno que la igualdad anterior conducía a la igualdad $2=6$ respondió ${ }^{i} \mathrm{Y}$ qué?»

Este alumno sí sabía que 2 no es igual a 6 . La resolución de esta contradicción, según Drouhard, es que para ese alumno la escritura simbólica del álgebra $\frac{10}{5}$ no designa el número 2, ni la escritura simbólica del álgebra $\underset{1}{6}$ designa el número 6.

Es decir, que, si las escrituras son privadas de esta designación, no se puede pedir que la igualdad respete designaciones ausentes. En consecuencia, la igualdad, en términos de transformaciones representa un papel idéntico al ya bien conocido en la aritmética: es decir, es el signo del resultado de una operación, no es ni simétri- 
co ni transitivo y «marca» el resultado de la transformación; asi, si se aplicara una transformación diferente, se obtendría otro resultado y, de todas maneras, la cuestión de la designación no es pertinente para el alumno.

En el ejemplo aquí tratado, si ni $\frac{10}{5}$ ni $\frac{6}{1}$ designan números, la igualdad: $\frac{10}{5}=\frac{6}{1}$ tiene pocas probabilidades de designar un valor de verdad (en particular, el valor «falso»); de ahí la elocuente respuesta del alumno que no percibe la contradicción: « Y qué?»

Según Drouhard, el no tomar en cuenta la denotación es característico de los sujetos que él Ilama autómatas formales, para quienes la cuestión de la validación de un resultado no se establece en términos del valor de verdad de la escritura obtenida (denotación), sino en términos de las reglas y los procedimientos. Para ellos un resultadoes válido porque se ha seguido un procedimiento correcto. De este modo, el alumno que está convencido de la simplificación $2=\frac{10}{5}=\frac{4+6}{4+1}=\frac{6}{1}=6$ entrará en un debate con el profesor (o experto) respecto a la contradicción $2=6$, que no será sino un intercambio de argumentos de autoridad (en un caso basados en el sentido del procedimiento y en el otro, en la denotación de las escrituras simbólicas); Drouhard llama a esto «diálogo de sordos».

El análisis de casos como el anterior, junto con sus consideraciones teóricas conducen a Drouhard a poner en juego cuatro componentes de las escrituras simbólicas del álgebra para hacer un replanteamiento etimológico sobre lo que significa comprender dichas escrituras. Su replanteamiento consiste en lo siguiente:

«Comprender tas Escrituras Simbólicas del Álgebra es tomar en cuenta de manera conjunta su sintaxis, su denotación, su sentido y su interpretación”. (Véase Drouhard 1992, cap. X, para una definición precisa de los términos que designan estos componentes).

Al igual que el modelo gramatical de Kirshner, el proyecto de Drouhard también restringe su análisis a ciertos aspectos del lenguaje matemático (algebraico), pero ello ha permitido, a su vez, empezar a tener un conocimiento más detallado y preciso del desempeño sintáctico en álgebra de los estudiantes. Aunque, como estos investigadores señalan, aún resta por hacer una gran cantidad de investigación básica para poder explotar adecuadamente en el ámbito didáctico los productos del trabajo realizado con esta perspectiva.

\section{NUEVAS PERSPECTIVAS DE ENSEÑANZA}

Aunque también con una preocupación teórica, existen investigaciones con una fuerte orientación didáctica, que tratan de aplicar en mayor o menor grado los conocimientos actuales sobre la psicolingǘstica al estudio del lenguaje matemático. Éste es el caso de la mayoría de los trabajos a los que se ha hecho alusión en el presente artículo, los cuales (como se ha tratado de señalar caso a caso) poseen un componente didáctico o, al menos, se advierte en ellos una preocupación en relación con las implicaciones que para la enseñanza pudieran llegar a tener sus respectivos planteamientos. Sin embargo, uno de los ejemplos más relevantes de esta vertiente es el trabajo de Pimm (1987), quien aborda tanto los aspectos verbales como los aspectos simbólicos de dicho lenguaje, en el contexto específico de su uso en ei satón de clases.

De las tres posibilidades de relacionar los términos matemáticas y lenguaje: el lenguaje de las matemáticas, matemáticas y lenguaje y las matemáticas como lenguaje, Pimm ubica su trabajo dentro de la tercera interpretación y declara como propósito principal de su obra construir las matemáticas en términos lingǘsticos. Un elemento básico en su trabajo es el uso de la metáfora, en el sentido de Lakoff y Johnson (1980): «la esencia de la metáfora consiste en comprender y experimentar una cosa en términos de la otra». En este caso, la relación metafórica se da entre la matemática y el lenguajc $y$, según lo expresa el propio Pimm, con este acercamiento se pueden formular cuestiones que de otro modo no habrían podido considerarse importantes (Pimm 1987, cap. IX).

Este autor hace una distinción importante de su propio tratamiento respecto del de aquellos autores que comparan el aprendizaje de la matemática con el aprendizaje de la lengua materna (tal es el caso de Gattegno, citado por el propio Pimm, y de Freudenthal, mencionado en una sección anterior de este escrito). Pimm se inclina por la comparación con el aprendizaje de una lengua extranjera, entre otras cosas, por las posibles consecuencias que traería realizar esta comparación en términos de las innovaciones en los métodos de enseñanza de los idiomas extranjeros. Pimm maneja un ejemplo muy significativo: el cambio en el centro de atención que ha ocurrido en la enseñanza de las lenguas extranjeras, el cual ha pasado de ser el idioma mismo a ser la competencia comunicativa en ese idioma.

Pimm afirma que, si la idea de la competencia comunicativa de las matemáticas ganara terreno, en el sentido de que el uso de éstas requiriera de ser consciente de las convenciones concretas que operan dependientes del contexto y de cómo tales convenciones influyen en lo que se comunica, «tal vez muchos más alumnos encontrarían que las matemáticas constituyen un estudio que compromete y que compensà) (Pimm 1987).

Pimm señala que la enseñanza de las matemáticas aún adolece de la misma deficiencia que la antigua enseñanza de las lenguas extranjeras, es decir, que tanto de parte de los profesores como de los alumnos hay una tendencia a centrar la atención en la forma de los enunciados matemáticos más que en las ideas expresadas por ellos. Esto es, el centro de atención está constituido por la notación simbólica y las cuestiones estructurales (la ortografía y la gramática).

Entre las implicaciones pedagógicas del trabajo de Pimm, se encuentra la necesidad de atender la comunicación 
oral en el aula, en correspondencia con una redefinición de los objetivos de la enseñanza de la matemática, a saber, devolver a los símbolos matemáticos su papel de medios, para reemplazar su papel de mensajes y substituir el aprendizaje de la matemática como un sistema abstracto regido por reglas (el cual resalta las formas escritas), por la adquisición de competencia comunicativa sobre determinados objetos, situaciones y fenómenos, con la consecuente importancia otorgada al lenguaje oral.

Aunque hoy en día, en algunas escuelas, se propicia el uso del lenguaje oral en clase por parte de los alumnos (por ejemplo, se organizan actividades conducentes a discusiones colectivas), en realidad la investigación sistemática del tipo de usos del lenguaje verbal en la clase de matemáticas ha sido muy escasa. En el libro de D. Pimm, se reseñan algunas investigaciones realizadas en esta dirección y se establecen distinciones importantes entre el habla matemática para comunicarse con el profesor, para hablar consigo mismo o para hablar a otros compañeros. Estas distinciones sugieren, a su vez, vertientes distintas de desarrollo de estudios que den cuenta del papel que representa el lenguaje oral en el aprendizaje y enseñanza de las matemáticas. Dichos estudios, a diferencia de algunos de los acercamientos teóricos descritos anteriormente, parecen tener posibilidades de aplicación de sus resultados a nivel de la enseñanza en un corto plazo. Ello permite hablar aquí, no sólo de nuevas perspectivas en la investigación, sino también de nuevas perspectivas en el campo de la enseñanza durante la presente década.

Para finalizar esta sección, quisiera aclarar que se ha omitido el importantísimo tema de los medios computacionales y los acercamientos de enseñanza e investigación de la inteligencia artificial, a pesar de que muchos trabajos desarrollados en este campo guardan una fuerte relación con la matemática concebida como lenguaje. La razón principal de tal omisión es que considero que, por su amplitud e importancia, el tema merece un tratamiento específico, que habría que desarrollar en un documento posterior.

\section{CONSIDERACIONES FINALES}

A lo largo de la presentación de una serie de trabajos de investigación que comparten la idea de ver la matemática como un lenguaje que va a ser enseñado, se ha tratado de ir scñalando aquellos elementos de cambio que afectan el qué y el cómo investigar el aprendizaje de la matemática, así como el qué y el cómo enseñar en la matemática escolar. Desde el momento en que buena parte de estos trabajos tienen como denominador común la incorporación de elementos de la psicolingǘstica o de Ja linguística a secas, es claro que las herramientas metodológicas de sus indagaciones toman ya ingredientes de la metodología utilizada en dichas disciplinas. Sin embargo, cabe señalar que es muy importante advertir las variantes de un enfoque a otro o de un trabajo a otro, pues así como el lenguaje matemático guarda diferencias sustanciales con las lenguas vernáculas, el conocimiento, la experiencia y jos métodos de investigación propios de estas últimas no pueden ser aplicados de manera directa al caso de la matemática.

Precisamente, las variaciones que se observan en este panorama de investigaciones, por un Iado, obedecen a que las bases teóricas de éstas se corresponden con diferentes corrientes o escuelas de la psicolinguística y, por otro, son una manifestación de la ausencia de un paradigma teórico para el estudio del sistema matemático de signos que abarque sus aspectos sintácticos, semánticos, pragmáticos y socioculturales.

Lo anterior implica que los acercamientos metodológicos se encuentran aún en evolución, así como que la delimitación de la problemática y de los objetos de estudio están en proceso de redefinición en las investigaciones recientes sobre la adquisición del Ienguaje matemático. Sin embargo, me atrevería, en este último caso de la definición del objeto de estudio, a tomar como punto referencial la distinción que hace Wertsh (1991) entre las unidades de análisis en un marco de la psicología piagetiana, constituidas por la adaptación y la interorización de esquemas conceptuales, a través de la interacción sujeto-objeto y las unidades de análisis en un marco psicolingüístico y sociocultural, constituidas por la acción y la interacción del medio ambiente con el funcionamiento mental humano, concebido éste como un producto de ese intercambio, a fin de subrayar, de nueva cuenta, diferencias básicas entre las aproximaciones conceptualista y psicolingüista. Contrastación que ha servido de eje para tratar de hacer convincente el argumento de que actualmente asistimos a una nueva era tanto en el terreno de la investigación en educación matemática como en la importante tarea de la enseñanza.

\section{NOTA}

* Ponencia presentada al IV Congreso intemacional sobre investigación en la didáctica de las ciencias y de las matemáticas, celebrado en Barcelona en septiembre de 1993. 


\section{REFERENCIAS BIBLIOGRÁFICAS}

BARUK, S, 1973. Échec et Maths. (Éd, du Seuil: París).

BOOTH, L., 1984. Algebra: Children's Strategies and Errors, (NFER-Nelson: Windsor).

BROUSSEAU, G., 1981. Problèmes de didactique des décimaux, Recherches en Didactique des Mathématiques, Vol. 2, pp. $37-128$.

CHOMSKY, N, 1957. Syntactic Structures. (Mouton: The Hague). Trad cast.: 1987, Estructuras sintácticas. (Siglo XXI: México).

CI.EMENT, J., 1982. Algebra word problem solutions: Thought processes underlying a common misconception, Journal for Research in Mathematics Education, Vol. 13, pp. 16-30.

COLLIS, K., 1975. A study of concrete and formal operations in school mathematics: A Piagetian viewpoint. (Australian Council for Educational Research: Melbourne).

CONROY,J., 1981. Learning language and mathematical structure in the infants school, Research in Mathematics Education in Australia, Vol. 2, pp. 203-212.

COOPER, M., 1984. The Mathematical «Reversal Error» and attempts to correct it, en B. Southwell, R. Eyland, $M$. Cooper, J. Conroy y K. Collis (eds.), Proceedings of the eighth International Conference for the Psychology of Mathematics Education, pp. 162-171.

DROUHARD, J. P., 1992. Les Écritures Symboliques del'Algèbre Élémentaire, Tesis Doctoral, Université Denis Diderot, París 7

FILLOY,E., 1988. Theoreticalaspects of PMEalgebra research. Documento interno. Institute of Education, University of London.

FILLOY, E, 1990. PME algebra research. A working perspective, Conferencia Plenaria, en G. Booker, P. Cobb y T. Mendecuti (eds.), Proceedings of the fourteenth International Conference for the Psychology of Mathematics Education, pp. II.1-II.33.

FILLOY, E., 1991. Cognitive Tendencies and Abstraction Processes in Algebra learning, en F. Furinghetti (ed.), Proceedings of the fifteenth International Conference for the Psychology of Mathematics Education, Vol. 2, pp. 48-55.

FII.LOY,E., 1993. Tendencias cognitivas y procesos de abstracción en el aprendizaje del Álgebra y de la Geometría, Enseñanza de las Ciencias, Vol. 11, pp. 160-166.

FILLOY, E. y ROJANO, T., 1989. Solving Equations: The Transition from Arithmetic to Algebra, For the Learning of Mathematics, Vol. 9, pp. 19-25.

FILLOY, E. y ROJANO, T., 1991. Translating from Natural Language to the Mathematical System of Algebraic Signs and viceversa, en R. Underhill (ed.), Proceedings of the thirteenth Conference of the North American Chapter of the International Group for the Psychology of Mathematics Education, Vol. 2, pp. 29-35.

HREGE, G., 1962. Estudios sobre semántica. (Ariel: Barcelona). FREGE, G., 1974. Escritos lógico-semánticos. (Tecnos: Madrid).
FREUDENTHAL, H., 1983. Didactical Phenomenology of Mathematical Structures. (Reidel Publishing Co.: Utrecht).

GOLDIN, G., 1987. Mathematical I,anguage and Problem Solving, en Janvier, C. (ed.), Problems of Representation in the Teching and learning of Mathematics. pp. 221-238. (Lawrence Erlbaum Associates Publishers: Hillsdale, NJ).

KAPUT, J, 1987. PME XI Algebra papers: A representational framework, en N. Bergeron, N. Herscovics y C. Kieran (eds.), Proceedings of the eleventh International Conference for the Psychology of Mathematics Education, pp. 345-354.

KAPUT, J,, 1989. Linking Representations in the Symbol Systems of Algebra, en Wagner, S. y Kieran, C. (eds.), pp. 167-194.

KIEREN, T., 1988. Personal Knowledge of Rational Numbers: Its Intuitive and Formal Developments, en J. Hiebert y $M$. Behr (eds.), Number Concepts and Operations in the Middle Grades. (Lawrence Erlbaum Associates Publishers/National Council of Teachers of Mathematics: Hilisdale, NJ/Reston, VA).

KIRSHNER, D., 1985. A Linguistic Model of Algebraic Symbol Skill. University of British Columbia. (No publicado).

KIRSHNER, D., 1987. Linguistic analysis of symbolic elementary algebra. Tesis doctoral. University of British Columbia.

KÜCHEMANN, D., 1981., Algebra, en Hart, K. (ed.), Children's Understanding of Mathematics, pp. 11-16. (Murray: London).

LABORDE, C., 1990. Language and Mathematics, en P. Nesher y J. Kilpatrick (eds.), Mathematics and Cognition, pp. 5369. (Cambridge University Press: Cambridge).

LAKOFF, G. y JOHNSON, M., 1980. Metaphors we live by. (University of Chicago Press: Chicago).

MATZ, M., 1982. Towards a process model for high school algebra errors, en D. Sleeman y J.S. Brown (eds.), Intelligent Tutoring Systems, pp. 25-50. (Academic Press: Londres).

NORMAN, F., 1987. A psycholinguistic perspective of algebraic language, en J. Bergeron, N. Herscovics y C. Kicran (eds.), Proceedings of the eleventh International Conference for the Psychology of Mathematics Education, Vol. 1, pp. 324 330 .

PIMM, D., 1987. Speaking Mathematically. (Routledge \& Kegan: Londres). Trad cast.: 1990, P. Manzano, El Lenguaje Matemático en el Aula. (Ministerio de Educación y Ciencia y Ediciones Morata: Madrid).

SKEMP, R. (ed), 1982. Understanding the symbolism of mathematics, edición especial de Visible language, Vol. XVI, núm. 3.

THOMPSON, P., 1989. Artificial Intelligence, Advanced Technology, and Learning and Teaching Algebra, en S. Wagner y C. Kieran (eds.), pp. 135-159.

VAN HIELE, P.M., 1987. A method to facilitate the finding of levels of thinking in Geometry by using the levels in Arithmetic. Learning and Teaching Mathematics - Issues for research and practise working, Syracusse University. 
VERGNAUD, G., I98I. Quelques Orientations Théoriques et Méthodologiques des Recherches Françaises en Didactique des Mathématiques, en $C$. Comiti y $G$. Vergnaud (eds.), Proceedings of the fifth International Conference of the Psychology of Mathematics Education, pp. 7-17.

VERGNAUD, G., 1987. About constructivism, en J. Bergeron, N. Herscovics y C. Kieran (eds.), Proceedings of the eleventh
International Conference of the Psychology of Mathematics Education, pp. 42-54.

WAGNER, S. y KIERAN, C., eds., 1989. Research Issues in the Learning and Teaching of Algebra. (Lawrence Eribaum Associates Publishers / National Council of Teachers of Mathematics: Hillsdale, NJ/Reston, VA).

WERTSH, J. V., 1991. Voices of Mind. A Sociocultural Approach to Mediated Action. (Harvester Wheatsheaf: Londres). 